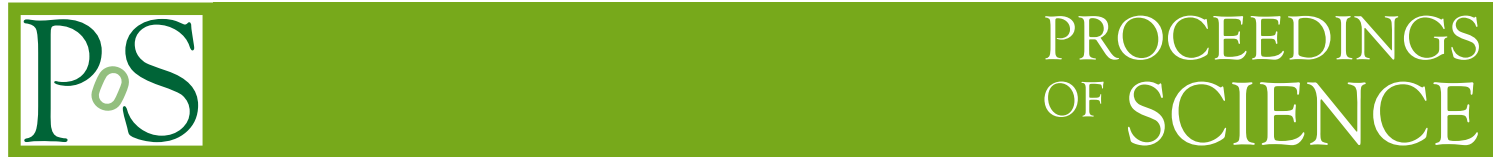

\title{
Strong Interactions for the LHC
}

\section{George T. Fleming*}

Yale University, P.O. BOX 208120, New Haven CT 06520-8120, USA

E-mail: George.Fleming@Yale.edu

Dynamical electroweak symmetry breaking (DEWSB) has been a viable option for the completion of the standard model for over thirty years. Precision electroweak studies indicate that the new strong interactions that break EW symmetry cannot be a scaled-up copy of QCD. Building viable models of DEWSB is difficult without a detailed understanding of such non-QCD gauge theories which still confine and break chiral symmetry. We review past difficulties of studying these theories using lattice methods and describe recent progress, focusing on the role of approximate infrared conformal symmetry.

The XXVI International Symposium on Lattice Field Theory

July 14-192008

Williamsburg, Virginia, USA

\footnotetext{
* Speaker.
} 


\section{INTRODUCTION}

The Large Hadron Collider (LHC) will soon probe physics at the TeV scale. Many theoretical models assume that phenomena at LHC energies involve strongly interacting systems (for reviews see [1-3].) Yet, theories of strong dynamics are very difficult to study from first principles. As a result, the predictions of many phenomenological models are affected by a large margin of uncertainty. Fortunately, recent advances in lattice gauge theory (LGT), paralleled by huge increases in available computational power, have made it possible to derive precise quantitative information for models based on strong dynamics by numerical simulation techniques. In the last year, several groups have begun aggressive calculational programs to expand our understanding of strongly interacting gauge theories that are hopefully unlike the very familiar quantum chromodynamics (QCD).

\section{Dynamical electroweak symmetry breaking}

The current standard model of electroweak interactions was first proposed in its essentially complete form by Weinberg in 1967 [4]. At the time, it was unclear whether this model was renormalizable and thus it was ignored for four years until its renormalizability was proven by 't Hooft [5]. Since then it has become the most successful theory of particle physics, withstanding more than three decades of testing with ever increasing precision. Despite the tremendous efforts of experimental particle physicists worldwide, two open questions remain. The first question is how to incorporate the phenomena of neutrino oscillations and will not be discussed further here. The second question is what are the nature of the Nambu-Goldstone bosons (NGBs) which are eaten to give mass to the electroweak bosons via the Higgs mechanism. This review will mostly focus on those mechanisms of electroweak symmetry breaking (EWSB) which involve new strong interactions and, thus, where LGT will be the preferred calculational tool for studying the dynamics of such variants of the electroweak theory.

The original proposal, still very much consistent with experimental data, is where a single Higgs doublet breaks electroweak symmetry and leaves behind a single, neutral scalar Higgs boson with a mass no more than a few hundred $\mathrm{GeV}$, which plays a critical role in making the electroweak theory viable at LHC energies. But theoretical advances in the 1970's have led to simple naturality arguments focused on the renormalization of the Higgs mass which conclude that this theory must break down at energies not more than a few $\mathrm{TeV}$. To make the breakdown scale higher and therefore clearly beyond the reach of the LHC would require some level of fine tuning of the minimal standard model. Rather than accept such careful fine-tuning, most theorists now conclude that some new physics described by a theory that extends beyond the standard model - a BSM theory - will manifest itself at LHC energies. Over the last three decades, a large number of candidate BSM theories has been developed. They must satisfy many stringent constraints in order to be viable, and this excludes many possibilities. Still, based on current experimental results it is not possible to select among them.

One of the earliest alternatives to the single Higgs doublet mechanism was proposed by Weinberg as well $[6,7]$ and is generally referred to as dynamical electroweak symmetry breaking (DEWSB), or sometimes technicolor [8] to emphasize the analogy with QCD. In the minimal 
technicolor model, a new strong force similar to QCD but operating at the TeV scale (three orders of magnitude larger than the QCD scale) plays a central role, driving electroweak symmetry breaking. In this model a host of new $\mathrm{TeV}$-scale resonances, analogous to the $\rho, \pi, \omega$, etc. of QCD, may be visible at the LHC. Unfortunately, a technicolor sector with the same low energy structure as QCD but scaled up to the electroweak scale is phenomenologically disfavored, as described below. However, this creates an opportunity for LGT specialists to survey the low energy landscape of spontaneously broken gauge theories for those with a sufficient number of NGBs and other low energy structure that may be quite different than QCD but favored by the phenomenology of DEWSB.

A much studied possibility in recent years is that space may have extra dimensions which are very small in size and therefore have not been observed up to now, but could be directly seen at LHC energies. In some extra-dimensional scenarios, the effective four-dimensional theory at LHC energies is weakly coupled, while in others the effective theory exhibits strong-coupling phenomena almost indistinguishable from technicolor theories. In fact, this similarity between some extradimensional models and technicolor models, typically referred to as "holography" or "Ads/CFT", has been employed by model builders to gain some insight into strong dynamics. This creates further opportunities for collaboration with LGT specialists to match these five-dimensional effective theories with their corresponding four-dimensional, UV-complete gauge theories. See review in these proceedings by E. Katz [9].

\section{WASN'T TECHNICOLOR RULED OUT A DECADE AGO?}

\subsection{The Peskin-Takeuchi $S$ parameter}

In the absence of any direct evidence for new particles that would reveal the physics of EWSB, three decades of effort starting with Veltman $[10,11]$ have gone into using precision electroweak parameters to limit the number of possible EWSB theories. A particularly useful pair of parameters, $S$ and $T$, were devised by Peskin and Takeuchi $[12,13]$ to place severe constraints on models of DEWSB. These constraints assume that changing the number of flavors $\left(N_{\mathrm{TF}}\right)$ or colors in $\mathrm{SU}\left(N_{\mathrm{TC}}\right)$ gauge theories mainly affects the counting of quark degrees of freedom. They must further assume important non-perturbative features remain unaltered: confinement, chiral symmetry breaking and the ordering of states in the hadronic spectrum. This will be called the scaled-up QCD hypothesis in this review. Assuming scaled-up QCD, $S$ in technicolor are estimated to be bounded by the very crude relation:

$$
S \gtrsim \frac{1}{6 \pi}\left[\frac{N_{\mathrm{TC}} N_{\mathrm{TF}}}{2}\right]
$$

The latest phenomenological constraints on $S$ and $T$, shown in Fig. 1, when combined with the naive scaling relation of Eq. (3.1) leads the Particle Data group to conclude: This rules out simple Technicolor models with many techni-doublets and QCD-like dynamics [1]. In other words, technicolor models with QCD-like dynamics and $N_{\mathrm{TC}}, N_{\mathrm{TF}} \lesssim 4$ are still consistent with, but somewhat disfavored by, precision electroweak experiments. Since the allowed range still includes QCD itself, it is important to directly calculate $S$ in lattice QCD and compare with estimates like Eq. (3.1) to show there are no underestimated systematic errors in the phenomenological approach. 


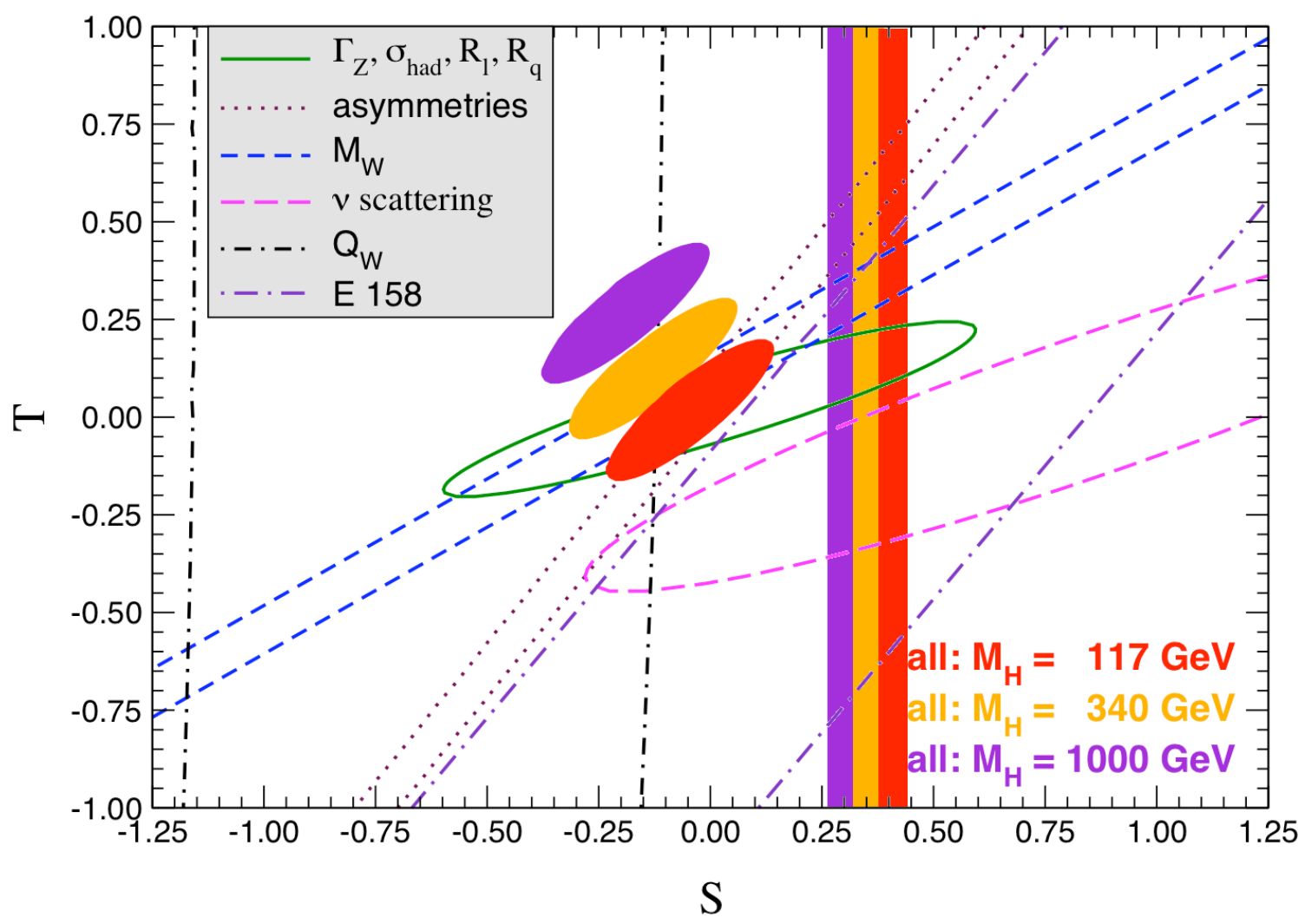

Figure 1: The shaded ellipses are the phenomenologically allowed regions for $S$ and $T$ at $68 \% \mathrm{CL}$, and show a strong correlation (87\%) between $S$ and $T$ [1,14]. The shaded vertical bands are the recently computed values of $S$ in technicolor with $N_{\mathrm{TC}}=3$ and $N_{\mathrm{TF}}=2$ at $68 \%$ CL [15]. The color of the shaded region indicates the reference value for the Higgs mass.

The basic observable that must be computed in QCD to estimate precision electroweak parameter $S$ in scaled-up QCD is the low energy part of the left-right current-current correlation function

$$
\Pi_{L R}^{\mu v}(q)=i g^{\mu v} \Pi_{L R}\left(q^{2}\right)+\left(q^{\mu} q^{v} \text { terms }\right) \equiv \int d^{4} x e^{-i q x}\left\langle J_{L}^{\mu}(x) J_{R}^{v}(0)\right\rangle
$$

The low energy part is given by the slope of the correlation function

$$
\Pi_{L R}^{\prime}(0)=\left.\frac{d}{d q^{2}} \Pi_{L R}\left(q^{2}\right)\right|_{q^{2} \rightarrow 0} .
$$

If we use the identity $J_{L, R}^{u}(x)=J_{V}^{\mu}(x) \pm J_{A}^{\mu}(x)$ then the low energy part is also given by $\Pi_{L R}^{\prime}(0)=$ $\left[\Pi_{V V}^{\prime}(0)-\Pi_{A A}^{\prime}(0)\right]$.

Once the current-current correlator has been computed, there are still several steps required to extract the precision electroweak parameters. In next-to-leading-order (NLO) chiral perturbation theory [16-19]

$$
\Pi_{L R}^{\mu v}(q)=\left[g^{\mu v}-\frac{q^{\mu} q^{v}}{m_{\pi}^{2}-q^{2}}\right] f_{\pi}^{2}+\left(q^{\mu} q^{v}-g^{\mu v} q^{2}\right)\left[\frac{1}{3}\left(1-\frac{4 m_{\pi}^{2}}{q^{2}}\right) \bar{J}\left(q^{2}\right)+\frac{1}{48 \pi^{2}}\left(\bar{l}_{5}-\frac{1}{3}\right)\right]
$$


where the goal is to determine $\bar{l}_{5}$. Note however that the first term has a pion pole contribution that must be included in any fit to the lattice results. The two-body phase space integral $J\left(q^{2}\right)$ arises from the two-pion intermediate state, leading to a cut starting at $q^{2}=4 m_{\pi}^{2}$.

The low energy constant $\bar{l}_{5}$ is logarithmically divergent in the chiral limit and must be cut off, introducing some scale dependence

$$
l_{5}^{r}(\mu)=\bar{l}_{5}+\log \frac{m_{\pi}^{2}}{\mu^{2}}
$$

where $\mu$ is some convenient UV scale, e.g. in QCD, typically $\mu=4 \pi f_{\pi}$ or $m_{\rho}$. In the context of DEWSB, precisely how the divergence is cut off will depend on the number of NGBs. Three of the NGBs will be cut off by the masses of the $W$ and $Z$ bosons when they are eaten. Extra pseudo Nambu-Goldstone bosons (PNGBs) will be problematic, if present, unless an additional mechanism, described in Sec. 3.2 is provided to give them masses. Once the PNGBs are massive, the should behave analogously to $K$ mesons in the chiral perturbation theory of QCD. Finally, the precision electroweak parameters like $S$ are defined to be zero in the minimal standard model with a single Higgs field. So, the standard model contribution must be subtracted, assuming some specific value for the Higgs mass. In summary, the low energy constant $\xi_{5}(\mu)$ computed in QCD is related to the precision electroweak parameter $S$ by

$$
S=\frac{1}{12 \pi}\left[l_{5}^{r}(\mu)+\log \frac{\mu^{2}}{m_{H}^{2}}-\frac{1}{6}\right]
$$

in the case $N_{\mathrm{TF}}=2$ with no PNGBs.

The first determination of $\bar{l}_{5}$ was made recently by the JLQCD collaboration [15]. The quoted value $L_{10}^{r}\left(m_{\rho}\right)=-5.2\left({ }_{-5}^{+7}\right) \times 10^{-3}$, converted in to the convention of Peskin and Takeuchi [13] by $l_{5}^{r}(\mu)=-192 \pi^{2} L_{10}^{r}(\mu)$, can be used with Eq. (3.6) provided $m_{\rho}$ is rescaled by identifying $F_{\pi}$ with the vacuum expectation value of the Higgs field, $v=2^{-1 / 4} G_{F}^{-1 / 2}=246 \mathrm{GeV}$. Taking $m_{\rho}=770 \mathrm{MeV}$ and the computed value of $F_{\pi}=87.3(5.6) \mathrm{MeV}$ from [20] yields $S=0.41\left(_{-4}^{+3}\right)$, $0.36\left({ }_{-4}^{+3}\right)$, and $0.30\left(_{-4}^{+3}\right)$ for $m_{H}=117,340$, and $1000 \mathrm{GeV}$, respectively. In Fig. 1, these allowed regions for $S$ are plotted along with the phenomenologically allowed regions for $S$ and $T$ at $68 \%$ CL. The disagreement between the two determinations a bit more than the two sigma level and essentially independent of the input Higgs mass.

So, the $S$ parameter can be used to place constraints on the number of technicolors $\left(N_{T C}\right)$ and techniflavors $\left(N_{\mathrm{TF}}\right)$ a technicolor theory may have when the underlying dynamics are like QCD. However, it is in fact a dynamical question whether a given theory is QCD-like as $N_{\mathrm{TC}}$ and $N_{\mathrm{TF}}$ are varied. If the dynamics of the theory are sufficiently different from QCD then the above estimates do not apply. In some walking theories (see Sec. 4.4 below) $S$ could be very small and possibly negative. So, the current phenomenological bounds on $S$ do not yet place stringent constraints on technicolor theories due to the lack of understanding how the dynamics of confining gauge theories change as the number of colors and flavors, or color representation of the fermions, are varied.

\subsection{Fermion masses and flavor changing neutral currents}

QCD-like technicolor theories with small $N_{\mathrm{TC}}$ and $N_{\mathrm{TF}}$ and no extra PNGBs cannot be ruled out alone by precision EW observables like the $S$ parameter. However, such a technicolor theory 
has only explained how the $W$ and $Z$ bosons have become massive through the Higgs mechanism. Technicolor alone does not explain how standard model fermions become massive nor how any additional NGBs beyond the three eaten during DEWSB become massive PNGBs. It seems natural to consider additional gauge interactions, called extended technicolor (ETC) [21, 22], that couple to both standard model fermions and technifermions. The ETC gauge symmetry will be broken at some higher scale $\Lambda_{\mathrm{ETC}}$ and at EW energies exchange of massive ETC gauge bosons will be effectively described by four fermion operators given schematically as

$$
\frac{(\bar{Q} Q)(\bar{Q} Q)}{\Lambda_{\mathrm{ETC}}^{2}}, \quad \frac{(\bar{q} q)(\bar{Q} Q)}{\Lambda_{\mathrm{ETC}}^{2}}, \quad \frac{(\bar{q} q)(\bar{q} q)}{\Lambda_{\mathrm{ETC}}^{2}} .
$$

where $Q$ 's are technifermions and $q$ 's are standard model fermions. When technicolor grows strong enough to break chiral symmetry and form $\langle\bar{Q} Q\rangle$ condensates, the first operator will give mass to uneaten PNGBs and the second operator will give mass to standard model fermions. However, the third operator is problematic because it gives rise to flavor changing neutral currents. In particular, experimental measurements of $K^{0}-\bar{K}^{0}$ mixing and $K_{L} \rightarrow \mu^{+} \mu^{-}$as well as upper limits on other rare processes require $\Lambda_{\mathrm{ETC}} \simeq 1000 \mathrm{GeV}$ [23].

Since ETC interactions will be highly suppressed at EW energies due to such a high ETC scale, it is natural to wonder whether standard model fermion masses can still be generated by this mechanism. For example, the mass of the strange quark will be given by $m_{s} \sim\langle\bar{Q} Q\rangle / \Lambda_{\mathrm{ETC}}^{2}$. In QCD, the chiral condensate is $\langle\bar{q} q\rangle / f_{\pi}^{3} \approx 25$. If $\langle\bar{Q} Q\rangle / v^{3} \sim 25$ as well, where $v=2^{-1 / 4} G_{F}^{-1 / 2}=$ $246 \mathrm{GeV}$, this leads to very light strange quarks: $m_{s} \sim 0.4 \mathrm{MeV}$. Thus, some new dynamics is required to produce modestly larger techniquark condensates than would naturally appear in a QCD-like theory. Walking is one such dynamical mechanism, described in Sec. 4.4. Even assuming a mechanism for enhancing condensates can be realized, flavor changing neutral currents still pose significant challenges for detailed models of DEWSB [23].

\section{FLAVOR DEPENDENCE OF SU(N) YANG-MILLS GAUGE THEORY}

In Sec. 4.1, we review what is known about the flavor dependence of $\mathrm{SU}(N)$ Yang-Mills theory from the perturbative expansion of the beta function. We also mention other non-perturbative and quasi-perturbative techniques for modeling flavor dependence. We focus on the question of whether theory has an infrared fixed point (IRFP) for a given number of flavors $N_{f}$. In Sec. 4.2, we review two decades of efforts to study this problem by determining the flavor dependence of the finite temperature phase transition. In Sec. 4.3, we review past and current efforts to understand the low energy dynamics of Yang-Mills by studying the non-perturbative running of the renormalized coupling.

\subsection{Yang-Mills Conformal Window}

The renormalization group describes the scale dependence of the renormalized coupling of Yang-Mills in a general scheme:

$$
L \frac{\partial}{\partial L} g(L)=\beta(g) \stackrel{g \rightarrow 0}{\sim} b_{0} g^{3}+b_{1} g^{5}+b_{2} g^{7}+\cdots
$$


In the asymptotic expansion $(g \rightarrow 0)$ the first two universal coefficients are independent of the scheme:

$$
b_{0}=-\frac{1}{(4 \pi)^{2}}\left(\frac{11}{3} N_{c}-\frac{2}{3} N_{f}\right), \quad b_{1}=-\frac{1}{(4 \pi)^{4}}\left[\frac{34}{3} N_{c}^{2}-\left(\frac{13}{3} N_{c}-\frac{1}{N_{c}}\right) N_{f}\right] .
$$

Of course, $b_{0}<0$ for $N_{f}<\frac{11}{2} N_{c}$ implies that $\mathrm{SU}\left(N_{c}\right)$ Yang-Mills gauge theory with relatively few number of flavors is asymptotically free.
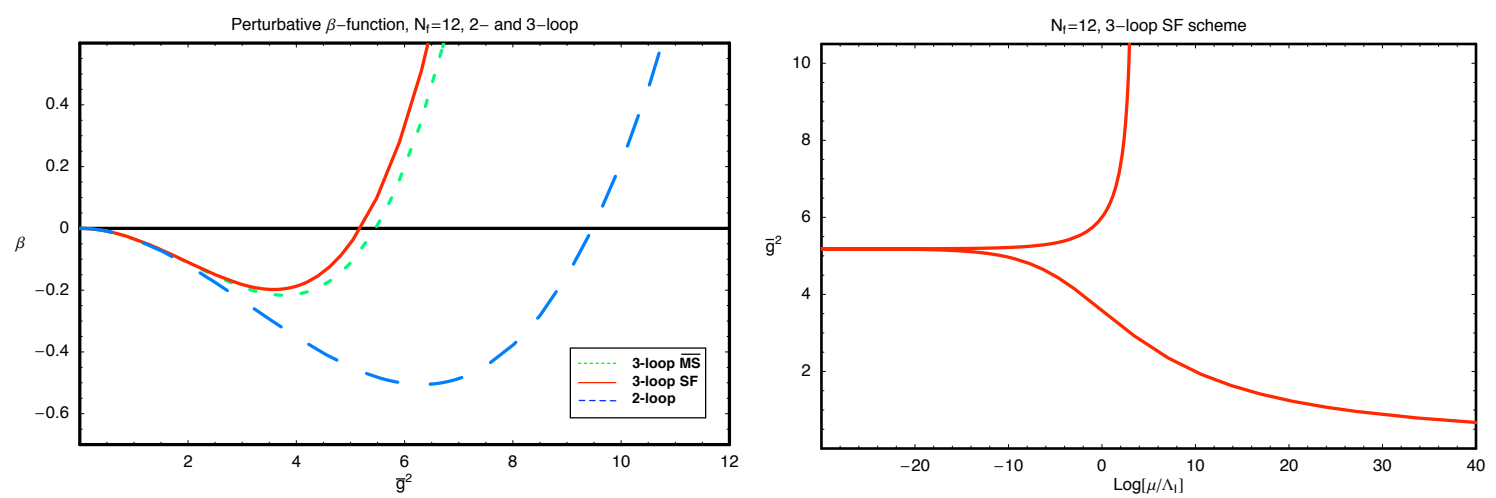

Figure 2: The left panel shows the two and three loop beta function in the Schrödinger Functional scheme with $N_{f}=12$ flavors [24]. The right panel shows the running of the coupling to the IR fixed point from both sides. The reference scale $\Lambda$ may be different on each branch.

For $N_{f} \lesssim \frac{11}{2} N_{c}$, the theory is still asymptotically free but the two loop term $b_{1}$ can cancel $b_{0}$ forming a perturbative infrared (IR) fixed point [25-27]. As $N_{f}$ steadily decreases from $\frac{11}{2} N_{c}$, the magnitude of the IR fixed point increases until higher order perturbative and even non-perturbative effects become significant, at which point even the definition of the renormalized coupling becomes scheme dependent. Regardless of the choice of scheme, the physics of the IR fixed point is made clear by the approximate conformal behavior of the theory at long distances. Such theories are said to be in the conformal window. Eventually, when $N_{f}$ is sufficiently small, the conformal window closes when the gauge interactions become strong enough to confine and/or spontaneously break chiral symmetry.

\subsection{Flavor Dependence of Finite Temperature Transition}

It would seem that lattice field theory techniques are ideally suited to determine the size of the conformal window in Yang-Mills. Yet, several efforts in the 1980's [28-32] and again in the 1990's [33-36] failed to produce a consistent picture of the zero temperature transition from a few flavors of confined quarks to many flavors of deconfined quarks in the non-Abelian Coulomb phase of the conformal window. This is in sharp contrast to the incredibly successful use of the lattice regulator to study the finite temperature transition of QCD during precisely the same period.

In Appendix A, we have summarized the relevant calculations from this period for staggered fermions with the unimproved SU(3) Wilson gauge action, with particular focus on $N_{f} \geq 3$ degenerate fermions. Although some studies exist with Wilson fermions [36] and staggered fermions with various forms of improvement, this forms the bulk of all such studies to determine the size 


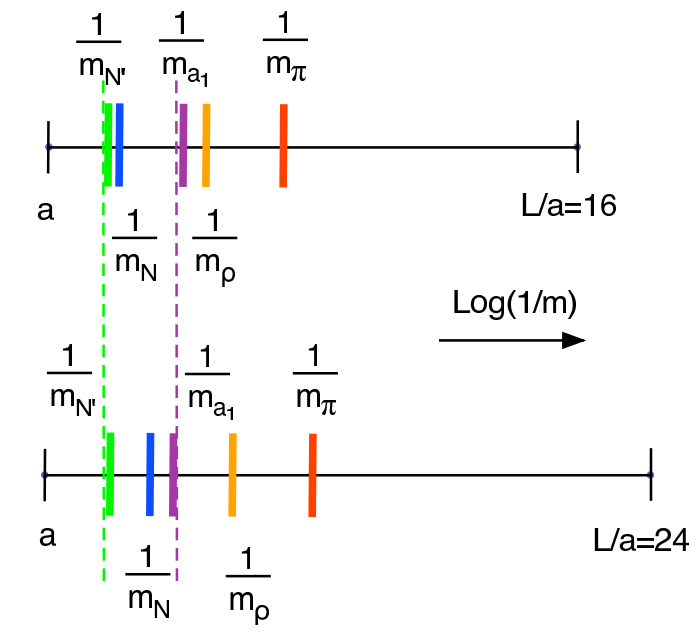

Source: Cheng-Zhong Sui, Ph.D. thesis, Columbia (2001)

Figure 3: Finite volume effects at fixed $\beta$ (thus fixed $a$ ) for $N_{f}=4$ staggered fermions disproportionally affect $\rho$ meson and $N$ nucleon and not their parity partners [37].

of the conformal window. The general trend of the data is clear: as the number of flavors increases, the critical temperature decreases relative to some dimensionful quantity computed at zero temperature, say $T_{c} / m_{\rho}$. Although each of the previous decades' efforts were encouraged by the theoretical, algorithmic and computational advances of the time, the failure to make progress appears related to the method of choice for defining the conformal window. In principle, one could study the finite temperature phase transition as the number of flavors increases and extrapolate this line of transitions to the critical number of flavors where the temperature vanishes. In practice, this proved to be quite challenging due to various difficulties in making the quarks light enough or the spatial box big enough (see Fig. 3).

Recently, two new groups have revived this approach. The Groningen-Frascati group is focused on eight and twelve flavors of improved (asqtad) staggered fermions [38-40]. The Columbia group is focused on eight flavors of unimproved staggered fermions with the DBW2 gauge action [41]. Both groups provide new evidence that SU(3) Yang-Mills with eight flavors is a confining theory with spontaneously broken chiral symmetry and a lattice spacing that vanishes as the bare coupling approaches zero. Apparently, the use of improved actions as well as more computational power was all that was needed to resolve some of the questions raised by the earlier work of the Columbia group at eight flavors [35]. Both groups are interested in applying their methods to study SU(3) Yang-Mills with twelve flavors where it is likely the theory is governed by an IRFP at low energies.

\subsection{Flavor Dependence of the Running Coupling}

In hindsight, the calculations of Damgaard et al. [33] and Heller [42, 43] of Yang-Mills with $N_{c}=3$ colors and $N_{f}=16$ flavors revealed the basic problem with the approach: in order to see chiral symmetry breaking on a finite temperature lattice with relatively few lattice sites in the temporal direction the bare lattice coupling must be made relatively strong. Using the renormalization 
group, this implies that the lattice spacing is relatively large and the temperature is relatively low. However, if the dynamics at the scale of the lattice spacing are strong enough, using the renormalization group to take the continuum limit may not be possible. In the 16 flavor case, the IR fixed point is completely understood in perturbation theory and no chiral symmetry breaking should occur. Yet, Damgaard et al. observed such a transition using the same techniques employed by other groups with smaller numbers of flavors. They surmised, and Heller later showed, that the chiral transition occurred along a renormalization group trajectory where the gauge dynamics becomes arbitrarily strong at short distances. In other words, there is no continuum limit for the apparently observed finite temperature transition because it occurs on the wrong side of the IR fixed point. This is shown in Fig. 2 for twelve flavors. Sixteen flavors is similar except the fixed point value for the running coupling is much smaller. The point is that the continuum limit doesn't exist if the coupling flows to the IR fixed point from above because the theory flows to strong coupling in the ultraviolet. Thus, it is reasonable to question the validity of the other aforementioned calculations since it is not at all clear that they are connected to the asymptotically free continuum limit by the renormalization group.

There are in fact several reasonable, non-perturbative definitions of the Yang-Mills running coupling which all coincide to two loops in perturbation theory. One example can be derived from the static quark potential $V(R)$. For an infinitely heavy quark-antiquark pair separated by a distance $R$ the force $F(R)$ felt by the pair is defined to be proportional to the renormalized coupling at the distance $R$

$$
\alpha_{Q \bar{Q}}(R)=\frac{g_{Q \bar{Q}}^{2}(R)}{4 \pi} \equiv \frac{1}{C_{F}} R^{2} F(R), \quad F(R)=\frac{\partial}{\partial R} V(R)
$$

For pure Yang-Mills $\left(N_{f}=0\right)$, the confining potential grows linearly at long distances so $\alpha_{Q \bar{Q}}(R) \stackrel{R \rightarrow \infty}{\sim}$ $R^{2}$. For a spontaneously broken theory $\left(N_{f}>0\right)$, the color flux tube responsible for the linear rise in the potential will eventually break when the static-light meson-antimeson state becomes energetically favored. Without some residual attractive force between the two static-light mesons, the renormalized coupling $\alpha_{Q \bar{Q}}(R)$ will rapidly vanish. In the conformal window, the force between static quarks is given by a non-Abelian Coulomb potential with the long distance coupling set by the IR fixed point.

In principle, the static quark potential is an ideal tool to search for the edge of the conformal window. In practice, the computational costs are such that the running of $\alpha_{Q \bar{Q}}(R)$ has only been computed out to confining distances in the quenched theory $[44,45]$. In a typical zero temperature lattice simulation with light dynamical quarks in a periodic box, there are four scales in the problem: the box size $L$, the light quark mass $m^{-1}$, the potential scale $R$ and the lattice spacing $a$. In general, all four scales should be widely separated, suggesting that $L>64 a$ at least, which is currently impossible given the available computational resources and the performance of the best available algorithms.

To make the calculation of the running coupling tractable in a practical lattice simulation given available computational resources, the coupling should be computed on a scale of $O(L)$ to eliminate the required scale separation $L \gg R$. A new non-perturbative scheme was presented at this meeting by M. Kurachi and E. Itou [46] where square Creutz ratios [47] calculated at a fixed fraction of the spatial box size can be used to define a renormalized coupling. This eliminates one of the separate 
scales needed in the static potential scheme and is therefore much more likely to be tractable in calculations with dynamical fermions. Further work is underway to eliminate the need for a nonzero quark mass using twisted boundary conditions.

Another non-perturbative definition of the running coupling is to perform calculations in a constant background chromoelectric field. The Schrödinger functional (SF) is the partition function describing the quantum mechanical evolution of a system from a prescribed state at time $t=0$ to another state at time $t=T$ in a spatial box of size $L$ with periodic boundary conditions [4850]. Dirichlet boundary conditions are imposed at $t=0$ and $t=T$ where $T$ is $O(L)$. They are chosen such that the minimum-action configuration is a constant chromo-electric background field of strength $O(1 / L)$. This can be implemented in the continuum [48] or with lattice regularization [24]. In either case, by considering the response of the system to small changes in the background field, a gauge invariant running coupling can be defined, valid for any coupling strength.

Heller had the original idea of studying the running of the SF renormalized coupling to look for flow towards an IR fixed point $[42,43]$. With very little computational effort, he clearly demonstrated that the $N_{f}=16$ chiral phase transition observed by Damgaard et al. [33] was triggered by strong dynamics at the UV scale and at long distances the coupling was flowing rapidly in the general direction of the very weak IR fixed point of $N_{f}=16$ flavors from above. Of course, to compute non-perturbatively the numerical value of such a weak fixed point would have required a great deal of computing power to statistically resolve it from zero.

In the conformal window, the coupling at the IR fixed point $g^{*}$ increases as the number of flavors decreases. So, for smaller $N_{f}$, while it may be desirable to continue to work on the "wrong" side of the IR fixed point due to the relatively rapid flow towards the fixed point there may be very little "room" in which to work without triggering a bulk lattice phase transition. On the asymptotically free side of the IR fixed point, the flow may be gradual and difficult to distinguish from lattice artifacts due to finite lattice spacing, but there will be confidence that an extrapolation to the continuum limit is still possible.

Deeper into the conformal window of $\mathrm{SU}\left(N_{c}\right)$ gauge theories, it is possible the IR fixed point $g^{*}$ may become sufficiently large that it is no longer well described by perturbation theory yet not strong enough to trigger spontaneous symmetry breaking. In SUSY QCD, this scenario leads to a non-trivial duality between the perturbative description defined in asymptotically free limit and a low energy description of new degrees of freedom that emerge at IR scales (see [51] for a review). There are arguments to suggest the IR fixed point of non-supersymmetric $\mathrm{SU}\left(N_{c}\right)$ gauge theories will remain perturbative throughout the conformal window [52]. So, a lattice calculation that could determine $g^{*}$ with sufficient accuracy to show disagreement with perturbation theory raises the possibility that something as interesting as Seiberg duality may occur near such a non-perturbative IR fixed point.

Recently, we presented the first non-perturbative evidence for the existence of an IRFP in SU(3) Yang-Mills with twelve flavors [53]. Additional evidence was presented that SU(3) with eight flavors shows no signs of an IRFP. Further details of the calculation were presented by Neil [54] at this meeting. The main observable computed was the running coupling in the SF scheme. Questions as to the scheme dependence of the conclusion are unavoidable and, thus, it is crucial to test this conclusion by other means. The work of the Columbia group and the GroningenFrascati group, already mentioned in Sec. 4.2, are important cross checks because they do rely 
on observables related to confinement and chiral symmetry breaking. We hope that the efforts of Kurachi, Itou and collaborators will soon result in a calculation of the running coupling in their scheme for twelve flavors to test the scheme-independence of the IRFP.

Finally, a calculation of the distribution of low-lying eigenvalues of the staggered Dirac operator was presented by Holland [55]. In this work, the authors present preliminary evidence that eigenvalue distributions for eight and twelve flavors of staggered fermions follow the predictions of random matrix theory for the epsilon regime $\left(M_{\pi} \ll L^{-1} \ll F_{\pi} \ll a^{-1}\right)$ of chiral perturbation theory. To determine if this result is evidence against the presence of an IRFP for twelve flavors, two questions must be resolved. First, what is $F_{\pi}$ and is the condition $L^{-1} \ll F_{\pi}$ satisfied on these configurations? Second, what are the expected eigenvalue distributions for twelve flavors if the low energy theory is governed by an IRFP, or if $F_{\pi} L \lesssim 1$, and how do they compare to the computed distributions? We believe the technique of epsilon-regime calculations can play an important role in future explorations of confining field theories. So, it is essential to continue these calculations until the apparent contradiction can be resolved.

\subsection{Walking Yang-Mills}

In the previous section, the goal was to identify the minimum number of flavors $N_{f}^{\text {crit }}$ needed to impede confinement and spontaneous chiral symmetry breaking in $\mathrm{SU}\left(N_{c}\right)$ gauge theory, thereby allowing the formation of an IR fixed point. Just below this critical number of flavors, it is plausible that Yang-Mills exhibits behavior at intermediate scales that is more like the physics of the nonAbelian Coulomb phase than of QCD. Of course, at very long distances, the theory must eventually confine otherwise it would be inside the conformal window. Such behavior is called "walking" because the renormalized coupling is expected to run much slower or "walk" when compared to QCD [56-60].

The main feature of walking theories that is of interest to model builders is that the pseudoscalar decay constant $f_{\pi}$ should be much smaller than the chiral condensate $\langle\overline{q q}\rangle$, in sharp contrast to QCD where the two quantities are of roughly the same order, e.g. $\langle\overline{q q}\rangle / f_{\pi}^{2} \approx 25$. In technicolor, for example, the scale of the Higgs VEV is set by the pseudoscalar decay constant but the mass scale of technihadrons, including any pseudo Nambu-Goldstone (NG) bosons are set by the much higher chiral condensate. If such a model were realized in Nature, no new resonances would be observed (including the Higgs) below the lowest technivector meson $\left(\rho_{r}\right)$ which is still too heavy to be observed directly at the Tevatron. See the review by Hill and Simmons [2] for details.

Another potentially interesting feature of a walking Yang-Mills gauge theory is that the spectrum of vector and axial-vector meson resonances may be significantly different than QCD, and perhaps even inverted [61]. Such an inverted spectrum would give rise to a negative $S$ parameter in contrast to the QCD-like behavior where $S$ increases with the number of flavors. Thus, without detailed knowledge about the low energy spectrum of a walking theory, precision electroweak constraints do not apply because they assume a QCD-like spectrum. 


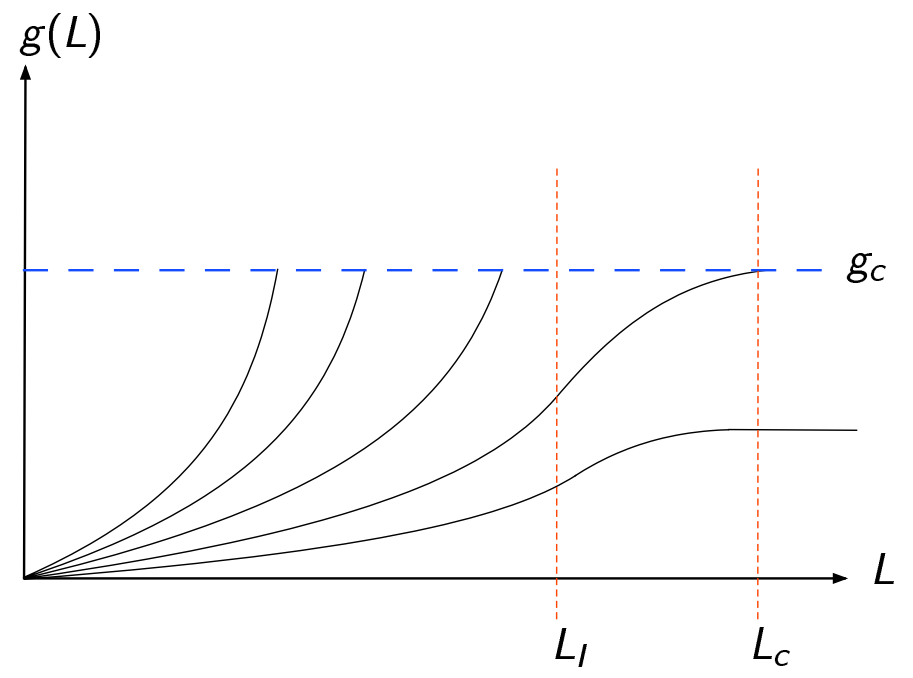

Figure 4: A cartoon of the dynamical scales of $\mathrm{SU}(N)$ Yang-Mills. $g(L)$ is a typical renormalized coupling. $g_{c}$ is the renormalized coupling required to trigger spontaneous chiral symmetry breaking ( $\mathrm{S} \chi \mathrm{SB}$ ). $L_{c}$ is the distance scale associated with $\mathrm{S} \chi \mathrm{SB} . L_{I}$ is the scale of the inflection point. The curves are various $N_{f}$ increasing from left to right. The rightmost curve is within the conformal window. A walking theory dynamically generates both scales $L_{I}$ and $L_{c}$.

\section{HIGHER FERMION REPRESENTATIONS IN SU( $N)$ YANG-MILLS GAUGE THEORY}

Recently, there has been a lot of interest in $\mathrm{SU}\left(N_{c}\right)$ Yang-Mills theories with $N_{f}$ fermions in higher representations of the gauge group. As with fermions in the fundamental representation, it is expected that the theories confine if $N_{f}<N_{f}^{\mathrm{c}}$, are governed by an IRFP if $N_{f}^{\mathrm{c}}<N_{f}<N_{f}^{\text {af }}$ (the conformal window), and asymptotic freedom is lost if $N_{f}>N_{f}^{\text {af }}$. Recent predictions of the extent of these conformal windows, shown in Fig. 5 [62], can now be tested by lattice methods.

There has been interest in the past [63-70] in the SU(3) theory with two Dirac flavors in the adjoint representation because of the possibility of two distinct finite temperature phase transitions. At higher temperatures, $\mathrm{SU}\left(2 N_{f}\right)$ chiral symmetry will break to $\mathrm{SO}\left(2 N_{f}\right)$ but the global $Z\left(N_{c}\right)$ center symmetry of the gauge action will remain unbroken until lower temperatures. This theory is apparently outside the conformal window although not much is known about the zero temperature, low energy effective description.

Two years ago, there were some preliminary calculations done by Catterall and Sannino [71] studying SU(2) Yang-Mills with $N_{f}=2$ flavors of adjoint fermions. In the past year, they have extended their work with new collaborators [72]. Two other groups have also presented new calculations for this theory and presented their work at this meeting [73-77]. It is reassuring to see a great deal of consistency amongst the groups as to the results of specific calculations. However, larger volume calculations are needed to bound the size of finite volume effects.

Two other groups also presented first results on SU(3) Yang-Mills with $N_{f}=2$ flavors of fermions in the two-index symmetric, or sextet, representation. DeGrand and Svetitsky [78-81] presented evidence using the Wilson fermion formulation that the low energy behavior of the the- 


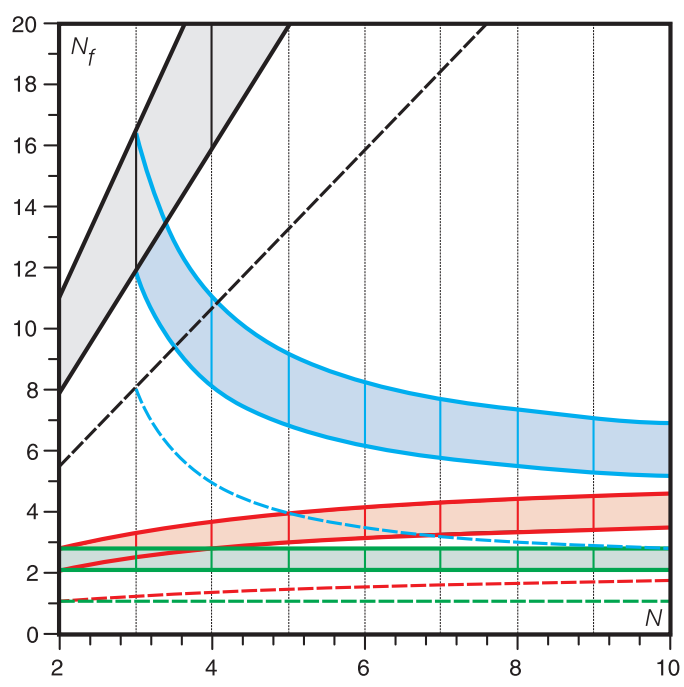

Figure 5: Zero temperature phase diagram for $\mathrm{SU}(N)$ Yang-Mills gauge theories with Dirac fermions in various color representations, taken from Ref. [62]. Representations from top to bottom are fundamental (grey), two-index antisymmetric (blue), two-index symmetric (red), and adjoint (green). Shaded regions are estimates of the conformal windows. Dashed curves indicate where the two-loop fixed point coupling diverges.

ory is governed by an IRFP on the basis of the running of the renormalized coupling in the SF scheme, similar to the case of $N_{f}=12$ flavors of fermions in the fundamental representation described in Sec. 4.3. Their results are quite interesting. Further calculations are done closer to the continuum limit would be welcome, perhaps for $L / a=16$ as was typical for QCD calculations done by the ALPHA collaboration. Nógrádi presented preliminary results [82] for an epsilon-regime calculation of eigenvalue distributions for the same theory using the overlap fermion formulation. We believe the same comments regarding the epsilon-regime calculation at twelve flavors apply here and look forward to their results with dynamical fermions.

Much has been made of the fact that the conformal window is likely to extend to much lower values of $N_{f}^{c}$ than is the case for the fundamental representation and that this has implications for the value of the $S$ parameter and the possibility of walking behavior. It is reasonable to expect that the low energy dynamics of these theories will be quite different than QCD and that naive estimates for the $S$ parameter are likely to be unreliable, so we should wait for a direct calculation of the $S$ parameter in these theories before drawing any conclusion about viability given the precision EW constraints.

Finally, walking dynamics are useful for model builders because it produces condensates $\langle\bar{Q} Q\rangle$ that are enhanced relative to their natural scale $F_{\pi}$ as described in Sec. 4.4. This allows the breaking of ETC gauge symmetry to be pushed to high scales to naturally suppress FCNCs while still allowing for reasonable masses for standard model fermions. We are unaware of any ETC model involving technifermions in higher representations. It would be interesting to see to what extent the walking mechanism will be necessary and/or useful in an ETC context should the LHC discover DEWSB by higher representation fermions. 


\section{ACKNOWLEDGMENTS}

We would like to thank the members of the Lattice Strong Dynamics (LSD) collaboration for their many useful discussions and careful reading of these proceedings: T. Appelquist, R. Babich, R. C. Brower, M. Cheng, M. A. Clark, M. Kastoryano, T. Luu, E. T. Neil, J. C. Osborn, C. Rebbi, D. Schaich, R. Soltz and P. M. Vranas. We would also like to thank the organizers and attendees of the Workshop on Lattice Gauge Theory for LHC Physics, May 2-3, 2008, Livermore, CA and the Workshop on Dynamical Electroweak Symmetry Breaking, 9-13 September 2008, Odense, Denmark, where there were many useful discussions that had impact on the remarks presented here.

\section{A. Flavor Dependence of Finite Temperature Transition}

Since the last comprehensive review of the flavor dependence of the finite temperature transition (including $N_{f} \geq 4$ ) was two decades ago [83], it seems appropriate to revise and extend the summary tables here for the bare critical coupling $\beta_{c} v s$. temporal extent $N_{t}$, and where possible, the critical temperature $T_{c}$ in dimensionful units. Until recently, the preferred action for these studies were the SU(3) Wilson gauge action and the unimproved Kogut-Susskind staggered fermion action. For $N_{f}$ not a multiple of four, some form of the rooting trick was applied.

Table 1: Summary of critical temperature calculations for SU(3) Yang-Mills with $N_{f}=0-18$ flavors, using the unimproved Wilson gauge action and Kogut-Susskind staggered fermion action.

\begin{tabular}{c|c|c|c|c|c|c}
\hline \hline$N_{f}$ & $N_{t}$ & $N_{s}$ & $a m$ & $\beta_{c}$ & $T_{c}(\mathrm{MeV})$ & refs \\
\hline \hline 0 & 4 & 16 & $\infty$ & $5.6908(2)$ & $290.35(14)$ & {$[84]$} \\
0 & 4 & $\infty$ & $\infty$ & $5.69254(24)$ & $291.54(16)$ & {$[85]$} \\
0 & 4 & $\infty$ & $\infty$ & $5.6925(2)$ & $291.52(14)$ & {$[84]$} \\
\hline 0 & 6 & 32 & $\infty$ & $5.8938(11)$ & $293.47(59)$ & {$[84]$} \\
0 & 6 & $\infty$ & $\infty$ & $5.89405(51)$ & $293.60(27)$ & {$[85]$} \\
0 & 6 & $\infty$ & $\infty$ & $5.8941(5)$ & $293.63(27)$ & {$[84]$} \\
\hline 0 & 8 & 32 & $\infty$ & $6.0609(9)$ & $292.01(42)$ & {$[84]$} \\
0 & 8 & $\infty$ & $\infty$ & $\approx 6.0625$ & $\approx 292.75$ & {$[84]$} \\
\hline 0 & 12 & 32 & $\infty$ & $6.3331(13)$ & $290.94(52)$ & {$[84]$} \\
0 & 12 & $\infty$ & $\infty$ & $\approx 6.3384$ & $\approx 293.09$ & {$[84]$} \\
\hline \hline 1 & 4 & 8 & 0.05 & $5.475(5)$ & & {$[86,87]$} \\
1 & 4 & 8 & 0.05 & 5.48 & & {$[88]$} \\
1 & 4 & 8 & 0.1 & $5.51(1)$ & & {$[86,87]$} \\
1 & 4 & 8 & 0.1 & $5.53(1)$ & & {$[89]$} \\
1 & 4 & 8 & 0.2 & $5.57(1)$ & & {$[86,87]$} \\
1 & 4 & 8 & 0.4 & $5.63(1)$ & & \\
\hline \hline
\end{tabular}




\begin{tabular}{|c|c|c|c|c|c|c|}
\hline \multicolumn{7}{|c|}{ Continued from previous page } \\
\hline$N_{f}$ & $N_{t}$ & $N_{s}$ & am & $\beta_{c}$ & $T_{\bar{c}}(\mathrm{MeV})$ & refs \\
\hline 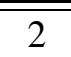 & 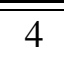 & $\overline{16}$ & 0.01 & $5.265(5)$ & 153.6(1.7) & [90] \\
\hline 2 & 4 & 8 & 0.0125 & $5.269(2)$ & $153.3(7)$ & [91] \\
\hline 2 & 4 & 12 & 0.0125 & $5.271(2)$ & $154.0(7)$ & [91] \\
\hline 2 & 4 & 8 & 0.025 & $5.286(2)$ & $150.8(6)$ & [91] \\
\hline 2 & 4 & 8 & 0.025 & 5.2875 & 151.3 & {$[92,93]$} \\
\hline 2 & 4 & 12 & 0.025 & $5.288(2)$ & $151.4(6)$ & [91] \\
\hline 2 & 4 & 16 & 0.025 & $5.291(1)$ & $152.4(3)$ & [90] \\
\hline 2 & 4 & 8 & 0.05 & $5.34(1)$ & $151.5(3)$ & [87] \\
\hline 2 & 4 & 8 & 0.05 & 5.32 & 146.5 & {$[92,93]$} \\
\hline 2 & 4 & 8 & 0.1 & $5.3825(25)$ & $138.8(4)$ & [87] \\
\hline 2 & 4 & 8 & 0.1 & 5.375 & 137.7 & {$[92,93]$} \\
\hline 2 & 4 & 8 & 0.1 & $5.3825(50)$ & $138.8(7)$ & [89] \\
\hline 2 & 4 & 12 & 0.1 & $5.376(3)$ & $137.8(4)$ & [89] \\
\hline 2 & 6 & 12 & 0.0125 & 5.415 & 144.1 & [94] \\
\hline 2 & 6 & 12 & 0.025 & 5.445 & 140.8 & [94] \\
\hline 2 & 6 & 12 & 0.025 & 5.4375 & 138.5 & {$[93]$} \\
\hline 2 & 6 & 12 & 0.05 & 5.47 & 125.4 & [93] \\
\hline 2 & 6 & 12 & 0.1 & 5.525 & 106.0 & [93] \\
\hline 2 & 8 & 16 & 0.004 & $5.43-5.53$ & $121-165$ & [95] \\
\hline 2 & 8 & 16 & 0.00625 & $5.475-5.5$ & $135-145$ & [96] \\
\hline 2 & 8 & 16 & 0.0125 & $5.54(2)$ & $151(9)$ & [97] \\
\hline 2 & 12 & 24 & 0.008 & $5.675(25)$ & $168(15)$ & [98] \\
\hline 2 & 12 & 24 & 0.016 & $5.775(25)$ & $186(13)$ & [98] \\
\hline 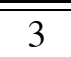 & 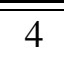 & 16 & 0.01 & $5.105(1)$ & & [99] \\
\hline 3 & 4 & 16 & 0.02 & 5.1235 & & {$[100]$} \\
\hline 3 & 4 & 8 & 0.025 & $\sim 5.1$ & & {$[101]$} \\
\hline 3 & 4 & 16 & 0.025 & 5.132 & & {$[90,99,100]$} \\
\hline 3 & 4 & 32 & 0.025 & 5.132 & & [100] \\
\hline 3 & 4 & 16 & 0.03 & 5.1396 & & {$[99,102]$} \\
\hline 3 & 4 & 16 & 0.0325 & 5.1396 & & [102] \\
\hline 3 & 4 & 16 & 0.035 & $5.1505(5)$ & & {$[100,102]$} \\
\hline 3 & 4 & 16 & 0.04 & 5.1593 & & {$[99,102]$} \\
\hline 3 & 4 & 8 & 0.1 & $5.25-5.3$ & & [103] \\
\hline 3 & 4 & 8 & 0.1 & $5.30(5)$ & & [104] \\
\hline 3 & 4 & 8 & 0.2 & $5.35(5)$ & & [104] \\
\hline 4 & 4 & 16 & 0.01 & 4.95 & & [105] \\
\hline 4 & 4 & 8 & 0.0125 & $4.919(6)$ & & [106] \\
\hline 4 & 4 & 8 & 0.025 & $4.94(1)$ & & [106] \\
\hline
\end{tabular}




\begin{tabular}{c|c|c|c|c|c|c}
\hline \hline \multicolumn{7}{c}{ Continued from previous page } \\
\hline \hline$N_{f}$ & $N_{t}$ & $N_{s}$ & $a m$ & $\beta_{c}$ & $T_{c}(\mathrm{MeV})$ & refs \\
\hline \hline 4 & 4 & 8 & 0.025 & $\sim 5.03$ & & {$[101]$} \\
4 & 4 & 8 & 0.025 & $4.983(3)$ & & {$[91]$} \\
4 & 4 & 16 & 0.025 & 4.99 & & {$[105]$} \\
4 & 4 & 8 & 0.0375 & $4.99(1)$ & & {$[106]$} \\
4 & 4 & 16 & 0.0375 & 5.02 & & {$[105]$} \\
4 & 4 & 16 & 0.05 & 5.04 & & {$[105]$} \\
4 & 4 & 8 & 0.1 & $5.05-5.15$ & & {$[107]$} \\
\hline 4 & 6 & 16 & 0.01 & 5.08 & & {$[105]$} \\
4 & 6 & 10 & 0.025 & $5.125(25)$ & & {$[106]$} \\
4 & 6 & 16 & 0.025 & 5.13 & & {$[105]$} \\
\hline 4 & 8 & 16 & 0.01 & $5.15(5)$ & $93(15)$ & {$[108]$} \\
\hline \hline 8 & 4 & 16 & 0.015 & $4.58(1)$ & & {$[35]$} \\
\hline 8 & 6 & 16 & 0.015 & $4.71(1)$ & & {$[35]$} \\
8 & 8 & 16 & 0.015 & $4.73(1)$ & & {$[35]$} \\
8 & 16 & 16 & 0.015 & $4.73(1)$ & & {$[35]$} \\
\hline \hline 10 & 4 & 8 & 0.1 & $4.70(10)$ & & {$[86]$} \\
10 & 4 & 8 & 0.2 & $4.85(5)$ & & {$[86]$} \\
10 & 4 & 8 & 0.4 & $5.15(1)$ & & {$[86]$} \\
10 & 4 & 8 & 0.6 & $5.35(5)$ & & {$[86]$} \\
10 & 4 & 8 & 1.0 & $5.60(10)$ & & {$[86]$} \\
\hline \hline 12 & 4 & 8 & 0.1 & $4.50(10)$ & & {$[86]$} \\
\hline \hline 16 & 6 & 16 & 0.1 & $4.11-4.13$ & & {$[33]$} \\
16 & 12 & 12 & 0.1 & $4.11-4.13$ & & \\
16 & 8 & 16 & 0.1 & $4.11-4.13$ & & \\
\hline \hline 18 & 4 & 8 & 0.1 & $4.25(15)$ & & \\
\hline \hline & & & & & \\
\hline
\end{tabular}

Table 2: Summary of scale setting calculations for SU(3) YangMills with $N_{f}=3-16$ flavors, using the unimproved Wilson gauge action and Kogut-Susskind staggered fermion action. Empirical interpolating functions are available for $N_{f}=0$ [109] and $N_{f}=2$ [110].

\begin{tabular}{c|c|c|c|c|c|c|c|c}
\hline \hline$N_{f}$ & $\beta$ & $m$ & $N_{s}$ & $N_{t}$ & $r_{0} / a$ & $a m_{\rho}$ & $a m_{N}$ & refs \\
\hline \hline 3 & 5.115 & 0.015 & 8 & 32 & $2.10(30)$ & & & {$[111,112]$} \\
3 & 5.1235 & 0.02 & 8 & 32 & $1.78(21)$ & & & {$[111,112]$} \\
\hline \hline
\end{tabular}




\begin{tabular}{c|c|c|c|c|c|c|c|c}
\hline \hline \multicolumn{7}{c}{ Continued from previous page } \\
\hline \hline$N_{f}$ & $\beta$ & $m$ & $N_{s}$ & $N_{t}$ & $r_{0} / a$ & $a m_{\rho}$ & $a m_{N}$ & refs \\
\hline \hline 3 & 5.132 & 0.025 & 8 & 32 & $1.38(17)$ & $1.300(14)$ & & {$[100,111,112]$} \\
3 & 5.1458 & 0.033 & 16 & 16 & & $1.387(38)$ & & {$[102]$} \\
3 & 5.151 & 0.035 & 8 & 32 & $1.51(19)$ & $1.313(11)$ & & {$[100,111,112]$} \\
3 & 5.3 & 0.01 & 16 & 32 & $3.9(1.0)$ & & & {$[111,112]$} \\
\hline \hline 4 & 5.15 & 0.01 & 16 & 24 & & $0.75(2)$ & $1.10(6)$ & {$[113,114]$} \\
\hline 4 & 5.2 & 0.01 & 12 & 24 & & $0.96(5)$ & $1.30(5)$ & {$[115,116]$} \\
4 & 5.2 & 0.025 & 12 & 24 & & $1.04(3)$ & $1.58(4)$ & {$[115,116]$} \\
4 & 5.2 & 0.05 & 12 & 24 & & $1.12(2)$ & $1.76(2)$ & {$[115,116]$} \\
4 & 5.2 & 0.075 & 12 & 24 & & $1.24(2)$ & $1.90(2)$ & {$[115,116]$} \\
\hline 4 & 5.35 & 0.01 & 12 & 24 & & $0.695(35)$ & $1.15(7)$ & {$[115,117]$} \\
4 & 5.35 & 0.01 & 16 & 24 & & $0.52(1)$ & $0.77(3)$ & {$[113,114,117]$} \\
4 & 5.35 & 0.025 & 12 & 24 & & $0.83(2)$ & $1.36(1)$ & {$[115,116]$} \\
4 & 5.35 & 0.05 & 12 & 24 & & $0.97(6)$ & $1.60(1)$ & {$[115,116]$} \\
4 & 5.35 & 0.075 & 12 & 24 & & $1.12(2)$ & $1.73(6)$ & {$[115,116]$} \\
\hline 4 & 5.4 & 0.01 & 16 & 32 & & $0.438(8)$ & $0.690(21)$ & {$[37,118]$} \\
4 & 5.4 & 0.01 & 24 & 32 & & $0.3742(44)$ & $0.5688(76)$ & {$[37,118]$} \\
4 & 5.4 & 0.015 & 16 & 32 & & $0.4736(59)$ & $0.7512(94)$ & {$[37,118]$} \\
4 & 5.4 & 0.02 & 16 & 32 & & $0.5008(28)$ & $0.7745(51)$ & {$[37,118]$} \\
4 & 5.4 & 0.02 & 24 & 32 & & $0.4872(26)$ & $0.7415(54)$ & {$[37,118]$} \\
\hline \hline 8 & 4.65 & 0.015 & 16 & 32 & & $0.522(7)$ & $0.872(10)$ & {$[35]$} \\
8 & 5.0 & 0.015 & 16 & 32 & & $0.484(7)$ & $0.807(7)$ & {$[35]$} \\
\hline \hline 16 & 4.125 & 0.1 & & & & $1.210(8)$ & $1.90(1)$ & {$[33]$} \\
16 & 4.25 & 0.1 & & & & $1.123(6)$ & $1.90(1)$ & {$[33]$} \\
16 & 4.375 & 0.1 & & & & $1.079(4)$ & $1.737(3)$ & {$[33]$} \\
\hline \hline
\end{tabular}

\section{References}

[1] R. S. Chivukula, M. Narain, J. Womersley, et al. (Particle Data Group), Phys. Lett. B667, 1 (2008).

[2] C. T. Hill and E. H. Simmons, Phys. Rept. 381, 235 (2003), hep-ph/ 0203079.

[3] K. Lane (2002), hep-ph/0202255.

[4] S. Weinberg, Phys. Rev. Lett. 19, 1264 (1967).

[5] G. ’t Hooft, Nucl. Phys. B35, 167 (1971).

[6] S. Weinberg, Phys. Rev. D13, 974 (1976). 
[7] S. Weinberg, Phys. Rev. D19, 1277 (1979).

[8] L. Susskind, Phys. Rev. D20, 2619 (1979).

[9] E. Katz, PoS LATTICE2008, 012 (2008).

[10] M. J. G. Veltman, Acta Phys. Polon. B8, 475 (1977).

[11] M. J. G. Veltman, Nucl. Phys. B123, 89 (1977).

[12] M. E. Peskin and T. Takeuchi, Phys. Rev. Lett. 65, 964 (1990).

[13] M. E. Peskin and T. Takeuchi, Phys. Rev. D46, 381 (1992).

[14] M. Grünewald et al. (ALEPH, DELPHI, L3, OPAL and SLD), Phys. Rept. 427, 257 (2006), hep-ex/0509008.

[15] E. Shintani et al. (JLQCD) (2008), 0806.4222.

[16] T. W. Appelquist and C. W. Bernard, Phys. Rev. D22, 200 (1980).

[17] A. C. Longhitano, Phys. Rev. D22, 1166 (1980).

[18] A. C. Longhitano, Nucl. Phys. B188, 118 (1981).

[19] J. Gasser and H. Leutwyler, Ann. Phys. 158, 142 (1984).

[20] H. Fukaya et al. (JLQCD), Phys. Rev. D77, 074503 (2008), 0711.4965.

[21] S. Dimopoulos and L. Susskind, Nucl. Phys. B155, 237 (1979).

[22] E. Eichten and K. D. Lane, Phys. Lett. B90, 125 (1980).

[23] T. Appelquist, M. Piai, and R. Shrock, Phys. Rev. D69, 015002 (2004), hep-ph/ 0308061 .

[24] A. Bode, P. Weisz, and U. Wolff (ALPHA), Nucl. Phys. B576, 517 (2000), hep-lat/ 9911018.

[25] W. E. Caswell, Phys. Rev. Lett. 33, 244 (1974).

[26] T. Banks and A. Zaks, Nucl. Phys. B196, 189 (1982).

[27] D. J. Gross and F. Wilczek, Phys. Rev. D8, 3633 (1973).

[28] R. V. Gavai, Nucl. Phys. B269, 530 (1986).

[29] J. B. Kogut, J. Polonyi, H. W. Wyld, and D. K. Sinclair, Phys. Rev. Lett. 54, 1475 (1985).

[30] N. Attig, B. Petersson, and M. Wolff, Phys. Lett. B190, 143 (1987).

[31] J. B. Kogut and D. K. Sinclair, Nucl. Phys. B295, 465 (1988). 
[32] S. Meyer and B. Pendleton, Phys. Lett. B241, 397 (1990).

[33] P. H. Damgaard, U. M. Heller, A. Krasnitz, and P. Olesen, Phys. Lett. B400, 169 (1997), hep-lat/9701008.

[34] S.-y. Kim and S. Ohta, Phys. Rev. D46, 3607 (1992).

[35] F. R. Brown et al., Phys. Rev. D46, 5655 (1992), hep-lat/9206001.

[36] Y. Iwasaki, K. Kanaya, S. Kaya, S. Sakai, and T. Yoshie, Phys. Rev. D69, 014507 (2004), hep-lat/0309159.

[37] C. Sui, Ph.D. thesis, Columbia University, New York (2001), UMI-99-98219, URL http: //wwwlib.umi.com/dissertations/fullcit?p9998219.

[38] A. Deuzeman, M. P. Lombardo, and E. Pallante (2008), 0804 . 2905.

[39] A. Deuzeman, M. P. Lombardo, and E. Pallante, PoS LATTICE2008, 060 (2008), 0810. 1719.

[40] A. Deuzeman, M. P. Lombardo, and E. Pallante, PoS LATTICE2008 (2008), 0810 . 3117.

[41] X.-Y. Jin and R. D. Mawhinney, PoS LATTICE2008, 059 (2008).

[42] U. M. Heller, Nucl. Phys. Proc. Suppl. 63, 248 (1998), hep-lat/9709159.

[43] U. M. Heller, Nucl. Phys. B504, 435 (1997), hep-lat/97050 12.

[44] S. Necco and R. Sommer, Nucl. Phys. B622, 328 (2002), hep-lat/ 0108008.

[45] S. Necco and R. Sommer, Phys. Lett. B523, 135 (2001), hep-ph / 0109093.

[46] E. Itou, M. Kurachi, et al. (2008), 0808.2875.

[47] M. Creutz, Phys. Rev. Lett. 45, 313 (1980).

[48] M. Lüscher, R. Narayanan, P. Weisz, and U. Wolff, Nucl. Phys. B384, 168 (1992), hep-lat/9207009.

[49] A. Bode et al. (ALPHA), Phys. Lett. B515, 49 (2001), hep-lat/ 0105003.

[50] S. Sint, Nucl. Phys. B421, 135 (1994), hep-lat/9312079.

[51] K. A. Intriligator and N. Seiberg, Nucl. Phys. Proc. Suppl. 45BC, 1 (1996), hep-th/ 9509066 .

[52] E. Gardi and G. Grunberg, JHEP 03, 024 (1999), hep-th/9810192.

[53] T. Appelquist, G. T. Fleming, and E. T. Neil, Phys. Rev. Lett. 100, 171607 (2008), 0712 . 0609.

[54] E. T. Neil, T. Appelquist, and G. T. Fleming, PoS LATTICE2008, 057 (2008). 
[55] Z. Fodor, K. Holland, J. Kuti, D. Nogradi, and C. Schroeder (2008), 0809 . 4890.

[56] B. Holdom, Phys. Rev. D24, 1441 (1981).

[57] B. Holdom, Phys. Lett. B150, 301 (1985).

[58] K. Yamawaki, M. Bando, and K.-i. Matumoto, Phys. Rev. Lett. 56, 1335 (1986).

[59] T. W. Appelquist and L. C. R. Wijewardhana, Phys. Rev. D35, 774 (1987).

[60] T. W. Appelquist and L. C. R. Wijewardhana, Phys. Rev. D36, 568 (1987).

[61] T. W. Appelquist and F. Sannino, Phys. Rev. D59, 067702 (1999), hep-ph/9806409.

[62] D. D. Dietrich and F. Sannino, Phys. Rev. D75, 085018 (2007), hep-ph/ 0611341.

[63] J. B. Kogut, J. Polonyi, H. W. Wyld, and D. K. Sinclair, Phys. Rev. Lett. 54, 1980 (1985).

[64] J. B. Kogut, Phys. Lett. B187, 347 (1987).

[65] F. Karsch and M. Lütgemeier, Nucl. Phys. Proc. Suppl. 73, 444 (1999), hep-lat/ 9809056 .

[66] F. Karsch and M. Lütgemeier, Nucl. Phys. B550, 449 (1999), hep-lat/9812023.

[67] M. Lütgemeier, Ph.D. thesis, Universität Bielefeld, Germany (1998), in german, URL http://www.physik.uni-bielefeld.de/theory/e6/doc/ dissertationen/luetgemeier.ps.gz.

[68] T. Schulze, Ph.D. thesis, Universität Bielefeld, Germany (2004), in german, URL http: //www.physik.uni-bielefeld.de/theory/e6/doc/dissertationen/ tschulze.ps.gz.

[69] J. Engels, S. Holtmann, and T. Schulze, Nucl. Phys. B724, 357 (2005), hep-lat/ 0505008 .

[70] J. Engels, S. Holtmann, and T. Schulze, PoS LAT2005, 148 (2006), hep- lat / 0509010.

[71] S. Catterall and F. Sannino, Phys. Rev. D76, 034504 (2007), 0705.1664.

[72] S. Catterall, J. Giedt, F. Sannino, and J. Schneible, JHEP 11, 009 (2008), 0807 . 0792.

[73] L. Del Debbio, M. T. Frandsen, H. Panagopoulos, and F. Sannino, JHEP 06, 007 (2008), 0802.0891 .

[74] L. Del Debbio, A. Patella, and C. Pica (2008), 0805 . 2058.

[75] A. Hietanen, J. Rantaharju, K. Rummukainen, and K. Tuominen, PoS LATTICE2008, 065 (2008), 0810.3722.

[76] L. Del Debbio, A. Patella, and C. Pica (2008), 0812 . 0570. 
[77] A. J. Hietanen, J. Rantaharju, K. Rummukainen, and K. Tuominen (2008), 0812 . 1467.

[78] Y. Shamir, B. Svetitsky, and T. DeGrand, Phys. Rev. D78, 031502 (2008), 0803.1707.

[79] B. Svetitsky, Y. Shamir, and T. DeGrand (2008), 0809.2885.

[80] T. DeGrand, Y. Shamir, and B. Svetitsky (2008), 0809.2953.

[81] T. DeGrand, Y. Shamir, and B. Svetitsky (2008), 0812 . 1427.

[82] Z. Fodor, K. Holland, J. Kuti, D. Nógrádi, and C. Schroeder (2008), 0809 . 4888.

[83] A. Ukawa, Nucl. Phys. Proc. Suppl. 10A, 66 (1989).

[84] G. Boyd et al., Nucl. Phys. B469, 419 (1996), hep-lat/9602007.

[85] Y. Iwasaki et al., Phys. Rev. D46, 4657 (1992).

[86] M. Fukugita, S. Ohta, and A. Ukawa, Phys. Rev. Lett. 60, 178 (1988).

[87] M. Fukugita and A. Ukawa, Phys. Rev. D38, 1971 (1988).

[88] R. V. Gavai, J. Potvin, and S. Sanielevici, Phys. Rev. D38, 3266 (1988).

[89] A. Irbäck, F. Karsch, B. Petersson, and H. W. Wyld, Phys. Lett. B216, 177 (1989).

[90] A. G. Vaccarino, Ph.D. thesis, Columbia University, New York (1991), UMI-92-09900, URL http://wwwlib.umi.com/dissertations/fullcit?p9209900.

[91] M. Fukugita, H. Mino, M. Okawa, and A. Ukawa, Phys. Rev. D42, 2936 (1990).

[92] S. A. Gottlieb, W. Liu, D. Toussaint, R. L. Renken, and R. L. Sugar, Phys. Rev. D35, 3972 (1987).

[93] S. A. Gottlieb, W. Liu, R. L. Renken, R. L. Sugar, and D. Toussaint, Phys. Rev. D38, 2245 (1988).

[94] C. W. Bernard et al. (MILC), Phys. Rev. D55, 6861 (1997), hep-lat/9612025.

[95] R. D. Mawhinney, Nucl. Phys. Proc. Suppl. 30, 331 (1993).

[96] S. A. Gottlieb et al., Phys. Rev. D55, 6852 (1997), hep-lat/9612020.

[97] S. A. Gottlieb et al., Phys. Rev. D47, 3619 (1993).

[98] C. W. Bernard et al., Phys. Rev. D54, 4585 (1996), hep-lat/9605028.

[99] S. Kaya et al. (JLQCD), Nucl. Phys. Proc. Suppl. 73, 459 (1999), hep-lat/9809102.

[100] X. Liao, Nucl. Phys. Proc. Suppl. 106, 426 (2002), hep-lat/ 0111013.

[101] R. V. Gavai, J. Potvin, and S. Sanielevici, Phys. Rev. Lett. 58, 2519 (1987). 
[102] F. Karsch, E. Laermann, and C. Schmidt, Phys. Lett. B520, 41 (2001), hep-lat/ 0107020 .

[103] R. V. Gavai and F. Karsch, Nucl. Phys. B261, 273 (1985).

[104] F. Fucito, S. Solomon, and C. Rebbi, Phys. Rev. D31, 1460 (1985).

[105] F. R. Brown et al., Phys. Lett. B251, 181 (1990).

[106] J. B. Kogut, E. V. E. Kovacs, and D. K. Sinclair, Nucl. Phys. B290, 431 (1987).

[107] J. Polonyi, H. W. Wyld, J. B. Kogut, J. Shigemitsu, and D. K. Sinclair, Phys. Rev. Lett. 53, 644 (1984).

[108] R. V. Gavai et al. $\left(M T_{c}\right)$, Phys. Lett. B241, 567 (1990).

[109] R. G. Edwards, U. M. Heller, and T. R. Klassen, Nucl. Phys. B517, 377 (1998), hep- lat/ 9711003.

[110] T. Blum, L. Karkkainen, D. Toussaint, and S. A. Gottlieb, Phys. Rev. D51, 5153 (1995), hep-lat/9410014.

[111] D. R. Nelson, G. T. Fleming, and G. W. Kilcup, Phys. Rev. Lett. 90, 021601 (2003), hep-lat/0112029.

[112] D. R. Nelson, Ph.D. thesis, The Ohio State University, Columbus (2002), hep-lat/0212009, URL http://wwwlib.umi.com/dissertations/ fullcit?p3081950.

[113] R. Altmeyer et al. $\left(M T_{c}\right)$, Nucl. Phys. B389, 445 (1993).

[114] K. D. Born, E. Laermann, R. Sommer, P. M. Zerwas, and T. F. Walsh, Phys. Lett. B329, 325 (1994).

[115] K. D. Born, E. Laermann, T. F. Walsh, and P. M. Zerwas, Nucl. Phys. Proc. Suppl. 9, 269 (1989).

[116] K. D. Born et al., Nucl. Phys. Proc. Suppl. 20, 394 (1991).

[117] E. Laermann et al. $\left(M T_{c}\right)$, Nucl. Phys. Proc. Suppl. 17, 436 (1990).

[118] R. D. Mawhinney, Nucl. Phys. Proc. Suppl. 83, 57 (2000), hep-lat/ 0001032. 